\title{
LA EDUCACIÓN POR EL ARTE: UNA APUESTA POR EL DESARROLLO INTEGRAL CON-Y A TRAVÉS DE LAS ARTES EN LA REFORMA EDUCATIVA PERUANA DE 1972
}

\author{
EDUCATION THROUGH ART: A COMMITMENT TO INTEGRAL \\ DEVELOPMENT WITH AND THROUGH THE ARTS IN THE PERUVIAN \\ EDUCATIONAL REFORM OF 1972
}

\author{
Lyscenia Durazo Córdova \\ Magíster en Educación \\ Profesora asociada \\ Pontificia Universidad Católica del Perú \\ ldurazo@pucp.pe
}

\begin{abstract}
Resumen: La Reforma Educativa peruana de 1972 marcó un hito en las propuestas educativas desde el Estado peruano al mostrar un profundo interés por incluir tendencias pedagógicas e ideológicas progresistas imperantes en su época, así como por fomentar el desarrollo del pensamiento crítico y utilizar métodos participativos de manera transversal a toda la propuesta curricular. En esta reforma, la línea de acción de Educación por el Arte del currículo de Educación Básica Regular se consolida como un área que aporta experiencias fundamentales relacionadas con capacidades psicomotrices, afectivo-sociales y cognitivas para el desarrollo integral del individuo. A partir de un análisis documental, la presente investigación analiza los elementos curriculares que conforman dicha línea de acción, tanto en el nivel macrocurricular como en el microcurricular. Se evidencia que esta reforma, centrada en el alumno, considera el arte y la educación artística como fines valiosos en sí mismos, y busca potenciar los aprendizajes del educando al explorar, imaginar, expresar, comunicar y crear con - y a través de- las artes mediante experiencias interdisciplinarias con el resto de las líneas de acción educativa.
\end{abstract}

Palabras clave: Reforma Educativa, currículo, educación artística, educación básica, educación integral

Resumo: A Reforma Educacional Peruana de 1972 marcou um marco nas propostas educacionais do Estado peruano por mostrar um profundo interesse em incluir as tendências pedagógicas e ideológicas progressistas vigentes em seu tempo, bem como por promover o desenvolvimento do pensamento crítico e a utilização de métodos participativos em um transversal a toda a proposta curricular. Nessa reforma, a linha de ação da Educação pela Arte do currículo da Educação Básica Regular se consolida como uma área que proporciona experiências fundamentais relacionadas às capacidades psicomotoras, afetivo-sociais e cognitivas para o desenvolvimento integral do indivíduo. A partir de uma análise documental, esta pesquisa analisa os elementos curriculares que

Fecha recepción: 1 de junio de 2021

Fecha aceptación: 6 de octubre de 2021 
compõem essa linha de ação, tanto no nível macrocurricular quanto microcurricular. É evidente que esta reforma, centrada no aluno, considera a arte e a educação artística como fins valiosos em si próprios e procura potenciar a aprendizagem do aluno ao explorar, imaginar, expressar, comunicar e criar com - e através - das artes. Através da interdisciplinaridade. experiências com as demais linhas de ação educativa.

Palavras chave: Reforma educacional, currículo, educação artística, educação básica, educação abrangente

Abstract: The Peruvian Educational Reform of 1972 marked a milestone in the educational proposals from the Peruvian State by showing a deep interest in including the pedagogical and progressive ideological trends prevailing in its time, as well as promoting the development of critical thinking and using participatory methods transversally to the entire curriculum proposal. In this reform, the line of action of Education through Art of the Regular Basic Education curriculum is consolidated as an area that provides fundamental experiences related to psychomotor, affective-social, and cognitive capacities for the integral development of the individual. Based on a documentary analysis, this research analyzes the curricular elements that make up this line of action, both at the macro-curricular and micro-curricular levels. It is evidenced that this reform, centered on the student, considers art and artistic education as valuable ends in themselves, and seeks to enhance learning by exploring, imagining, expressing, communicating, and creating with-and through - the arts through interdisciplinary experiences with other lines of educational action.

Keywords: Educational Reform, curriculum, arts education, elementary school, integral education

\section{INTRODUCCIÓN}

La propuesta de la Reforma Educativa peruana de 1972, llevada a cabo bajo el Gobierno Revolucionario de la Fuerza Armada (GRFA), al mando del general Juan Velasco Alvarado, mostró un gran interés por incorporar las tendencias pedagógicas e ideológicas progresistas imperantes en su época, así como por favorecer el desarrollo de habilidades para el estudio crítico y el uso de métodos participativos en pro del desarrollo integral del educando. A partir del informe presentado por la Comisión para la Reforma Educativa1, la actividad artística empezó a considerarse necesaria en todas las acciones educativas para el desarrollo pleno del individuo, y la creatividad se consideró el motor de todas las formas de comunicación y de realización pedagógica del sistema educativo.

\footnotetext{
${ }^{1}$ La Comisión para la Reforma Educativa fue conformada con el fin de elaborar un diagnóstico sobre la sociedad peruana para diseñar las propuestas educativas de la Reforma. Estuvo integrada por más de 100 miembros, entre militares e intelectuales, que concordaban con la necesidad de transformar el sistema educativo. La comisión culminó con el libro Reforma de la Educación Peruana; Informe General (Ministerio de Educación, Gobierno del Perú, 1970) y con más de 5000 fichas que derivarían posteriormente en la promulgación de la Ley General de Educación № 19326 (Ministerio de Educación, Gobierno del Perú, 1972).
} 
En esta reforma, la línea de acción de Educación por el Arte se constituye como un eje fundamental para la formación integral y para el desenvolvimiento completo del educando en la sociedad. Estas características la convierten en un objeto de estudio de gran interés, ya que, a través de sus elementos curriculares, surge la posibilidad de comprender de qué manera se concibió y se estructuró la propuesta curricular para alcanzar tales fines. Permite también profundizar en el recorrido de la educación artística en el sistema educativo peruano y conocer algunos de los abordajes que las artes han tenido en la educación básica. De esta manera, es posible identificar los aspectos epistemológicos, metodológicos y teóricos que han fundamentado las propuestas de educación artística en el sistema educativo y que pueden tener vigencia para los diseños curriculares de hoy en día.

El objetivo de la presente investigación es analizar los elementos curriculares de la Educación por el Arte en el currículo de Educación Básica Regular (EBR) de la Reforma Educativa de 1972 a partir de dos dimensiones desarrolladas específicamente para este análisis: la dimensión macrocurricular y la microcurricular. La investigación es de tipo documental y se desarrolla con el método de investigación documental bajo el enfoque cualitativo. Se emplea la técnica de análisis de documentos en cinco textos de carácter oficial y prescriptivo a partir de categorías y subcategorías relacionadas directamente con los objetivos de la investigación. Para el recojo de información, se diseñaron fichas individuales por cada subcategoría y para cada documento de análisis, las cuales detallan su ubicación exacta en el texto original y posibles observaciones sobre las citas textuales. Finalmente, con el objetivo de orientar el análisis, se diseñó una propuesta teórica general del currículo y una guía de análisis, las cuales permitieron trabajar sobre una estructura sólida de conceptualización del currículo.

\section{MARCO DE LA INVESTIGACIÓN}

\section{La educación artística y sus diversos enfoques}

En las propuestas curriculares que se observan alrededor del mundo, la educación artística es objeto de distintos enfoques y tendencias. Es decir, existen diferentes formas de concebir tanto los contenidos como la función de la educación artística en la educación básica, debido a que esta se encuentra determinada por el contexto sociocultural donde se implemente la propuesta curricular. En la actualidad, el mayor desafío que encontramos en la educación artística es la falta de criterios generales con relación a las disciplinas artísticas que debieran estructurar el área, o sobre los contenidos y los objetivos de aprendizaje que se pretenden alcanzar a través de las artes (Organización de Estados Iberoamericanos para la Educación, la Ciencia y la Cultura, 2014). En sí, cada propuesta curricular presenta un abordaje y una perspectiva de la educación artística diferentes. No obstante, en la mayoría de los casos existe un acuerdo sobre la importancia de la educación artística para la formación integral y el desarrollo pleno del individuo. 
En la Hoja de Ruta para la Educación Artística de la Organización de las Naciones Unidas para la Educación, la Ciencia y la Cultura (2006), se menciona que los campos o disciplinas que integran la educación artística deben ser considerados dentro de una clasificación siempre cambiante y nunca excluyente, y que comúnmente se puede incluir a las "artes escénicas (danza, teatro, música, etc.), la literatura y la poesía, la artesanía, el diseño, el arte digital, la narración, el patrimonio, las artes visuales y, finalmente, el cine, la fotografía y los medios" (p. 5) como disciplinas artísticas que la componen. A este respecto, en una investigación sobre el papel de las artes en la educación en 60 países, Bamford (2009, como se citó en Giráldez y Palacios, 2014) concluye que las disciplinas que conforman el área de educación artística generalmente son las artes plásticas, las artes visuales, la música, la expresión corporal, la danza y el teatro.

En el caso de la presente investigación, y de acuerdo con la propuesta curricular de la Reforma, se entiende por educación artística al campo del conocimiento conformado por distintas disciplinas artísticas, tales como la música, el teatro, la expresión corporal y las artes plásticas, las cuales buscan el desarrollo de capacidades vinculadas con el arte, la transmisión de la cultura y el desarrollo integral del individuo en el nivel básico de la educación escolar. El nombre de este campo del conocimiento en el currículo de la Reforma Educativa es Educación por el Arte, denominación que enmarca un claro enfoque de las artes en la educación.

En su estudio, Bamford (2009) plantea también dos posibles enfoques conceptuales sobre la educación artística a partir de cómo se abordan las artes en la educación, del concepto que se tiene de ellas en el currículo y de las prácticas de la misma educación artística: 1) la educación para el arte, la cual supone transmitir las prácticas y principios de las disciplinas artísticas para estimular la conciencia crítica y la sensibilidad para construir identidades culturales; y 2) la educación por el arte, en la que se considera al arte como un vehículo de aprendizaje de otras materias y un medio para conseguir resultados educativos más generales ${ }^{2}$ (Bamford, 2009).

A diferencia de la educación para el arte, la cual pretende formar futuros artistas y continuar con la tradición de formación artística profesional, en la educación por el arte las artes son de gran utilidad para la articulación de otras materias del plan de estudios y estas se pueden emplear de manera transversal dentro del currículo escolar. A este respecto, Jiménez, Aguirre y Pimentel (2009) afirman que, a partir del enfoque de educación por el arte, se han derivado diversas propuestas educativas que pretenden estimular la libertad de expresión, el desarrollo de la reflexión crítica y la tolerancia a través de las diversas expresiones artísticas.

Según Bamford (2009), es más habitual encontrar el enfoque de educación para las artes en la educación secundaria, mientras que la educación por el arte se encuentra frecuentemente en la educación primaria. Sin embargo, es fundamental tener en cuenta que ambos enfoques "son necesarios para aprovechar al máximo el potencial de cada niño y niña",

\footnotetext{
${ }^{2}$ En la bibliografía, también es posible encontrar como sinónimo de educación para el arte a la educación en el arte y como sinónimo de educación por el arte a la educación a través de las artes.
} 
y que estos son conceptos interdependientes que pueden coexistir dentro de un mismo currículo para que sus efectos positivos puedan visibilizarse en la educación (Bamford, 2009, p. 83).

\section{El currículo y sus elementos}

La pluralidad de significados que pueden encontrarse sobre el término currículo, así como la complejidad de dicho concepto, demandan en este estudio un claro posicionamiento con respecto a su definición y a la acepción que se usará durante la presente investigación. En primera instancia, se recurre al concepto de Ruiz (2005/2013), quien plantea que por currículo se puede entender

al conjunto de objetivos, competencias básicas, contenidos, métodos y estrategias de intervención didáctica y organizativa, así como las decisiones que ha de tomar el conjunto de profesores de un centro educativo sobre el modelo de evaluación y la aplicación de sus criterios, en función de las metas educativas que intentan alcanzar (p. 27).

Es decir, el currículo contiene elementos instrumentales que viabilizan la puesta en práctica dentro del aula y que concretan el proceso de enseñanza-aprendizaje. Son, tal como su nombre lo indica, los elementos que instrumentalizan el currículo en el curso de su aplicación y desarrollo. No obstante, de quedarnos exclusivamente con esta descripción, podríamos ver solo parcialmente la complejidad del currículo y correríamos el riesgo de no advertir sus múltiples dimensiones. Precisamos, por tanto, entender el currículo en el amplio espectro de su proceso y considerar que se erige también como

el eslabón entre la cultura y la sociedad exterior a la escuela y la educación, entre el conocimiento o la cultura heredados y el aprendizaje de los alumnos, entre la teoría (ideas, supuestos y aspiraciones) y la práctica posible, dadas unas determinadas condiciones (Gimeno Sacristán, 1988, p. 34).

Claramente, el currículo existe en más de una dimensión, ya que, además de estar integrado por los aspectos instrumentales que se desarrollan directamente en el aula y a través del docente en situaciones concretas, atañe asimismo a aspectos sociales, culturales y supuestos teóricos que permean toda la propuesta curricular. En ese mismo sentido, Tanner y Tanner (1980, como se citaron en Gimeno Sacristán y Pérez Gómez, 1992) afirman que existen dos dimensiones presentes en todo currículo: la dimensión macrocurricular y la dimensión microcurricular. Cada una de estas dimensiones se desarrolla, a su vez, en un nivel curricular específico y engloba elementos que, en conjunto, conforman la totalidad del currículo.

Estos niveles curriculares se clasifican a partir del grado de concreción del currículo en su proceso de desarrollo, así como en la toma de decisiones por parte de los actores involucrados en este proceso. De acuerdo con Ruiz (2005/2013) y Cantón Mayo y Pino-Juste (2011), se puede encontrar un primer nivel de concreción que corresponde al diseño del 
currículo por las administraciones educativas y a la elaboración de un diseño curricular base con planteamientos generales en torno a sus fundamentos. El segundo nivel de concreción compete a los centros educativos y se recoge en la planificación a mediano plazo del proyecto educativo (Cantón Mayo y Pino-Juste, 2011) y, finalmente, el tercer nivel de concreción se refiere a las programaciones de aula que el docente lleva a cabo directamente con sus alumnos. Generalmente, este se recoge en unidades didácticas estructuradas para cada área de conocimiento.

En tal sentido, es posible considerar la dimensión macrocurricular como aquella que acontece en el primer nivel de concreción del currículo, esto es, la dimensión que tiene lugar dentro de los procesos que suceden en el ámbito político administrativo y que son generalmente de carácter prescriptivo. Corresponde al punto de partida de la planificación y vida del currículo, y se refiere concretamente a las disposiciones jurídicas generales, a sus bases teóricas, a la estructura general del currículo dentro del sistema educativo y a las orientaciones curriculares generales. Los elementos que integran la dimensión macrocurricular son aquellos que tienen un carácter orientador y determinan la intencionalidad de las acciones educativas (Díaz, Persico, Del Mastro y Ferrazzi, 1992). Por tal motivo, se consideran elementos orientadores el diagnóstico educativo, las ideas rectoras, los perfiles3 y los objetivos generales (Díaz et al., 1992; Gonzales, García, Saco y Reátegui, 1987).

En lo que respecta a la dimensión microcurricular, se consideran los aspectos curriculares que se producen en el segundo y tercer nivel de concreción y que se desenvuelven dentro de la institución escolar y el aula. Esta dimensión se refiere específicamente a los componentes curriculares que traducen y concretan las orientaciones del currículo a través de los procesos pedagógicos. Es una dimensión que se encuentra estrechamente relacionada con la didáctica, la práctica educativa y el tratamiento de cada curso en particular (Gimeno Sacristán y Pérez Gómez, 1992). Los elementos que integran la dimensión microcurricular son, por lo tanto, los objetivos específicos, los contenidos, las actividades, los recursos y la evaluación (Díaz et al., 1992; Gonzales et al., 1987).

La propuesta teórica que se ha diseñado para el presente análisis permite diferenciar elementos orientadores e instrumentales, y tiene como base cuatro planteamientos: 1) el modelo conceptual Dimensiones Fundamentales del Currículo desarrollado por Gonzales et al. (1987); 2) la reconceptualización del mismo modelo elaborada por Díaz et al. (1992); 3) los aportes con relación a los niveles del currículo de Tanner y Tanner (1980, como se citaron en Gimeno Sacristán y Pérez Gómez, 1992); y 4) los niveles de concreción refrendados por Ruiz (2005/2013) y Cantón Mayo y Pino-Juste (2011). La terminología empleada y las relaciones entre los diversos elementos se derivan de las diversas clasificaciones y planteamientos propuestos por dichos autores (véase la Figura 1).

${ }^{3}$ Gonzales et al. (1987) hacen referencia a dos tipos de perfiles: educativos o de salida, y fácticos o de entrada. 


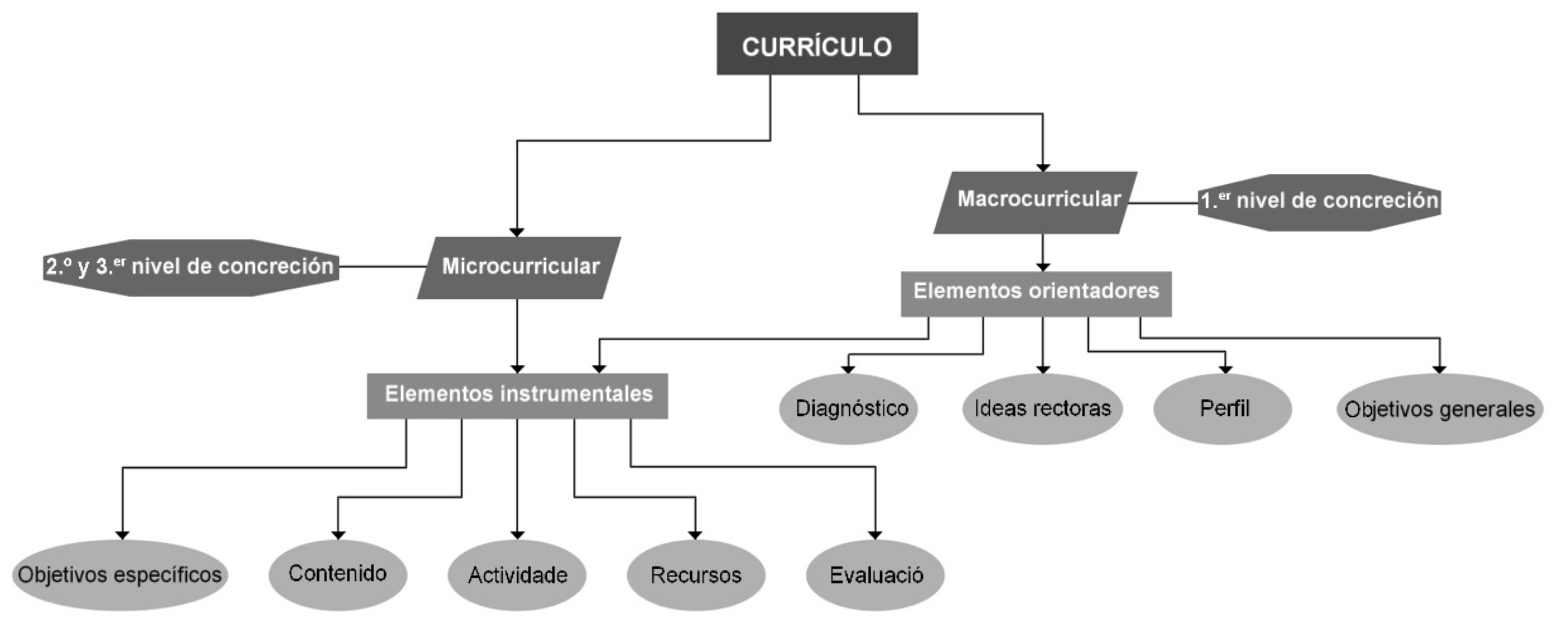

Figura 1. Propuesta teórica de análisis general de niveles curriculares y elementos del currículo. Adaptada de Diseño y desarrollo del currículum, por I. Cantón Mayo y M. Pino-Juste (Coords.), 2011, Alianza Editorial; de "Desarrollo del currículo de los centros educativos: análisis de los elementos orientadores e instrumentales", por C. Díaz $\quad$ Bazo et al., 1992, Educación, 1(1), (http://revistas.pucp.edu.pe/index.php/educacion/article/view/4402); de Tanner y Tanner, 1980, como se citaron en Comprender y transformar la enseñanza, por J. Gimeno Sacristán y A. I. Pérez Gómez, 1992, Morata; de Lineamientos básicos para el trabajo curricular. Documento de trabajo, por Gonzales et al., 1987, CISE-PUCP; y de Teoría del currículum: diseño, desarrollo e innovación curricular, por J. M. Ruiz, 2005/2013, Editorial Universitas.

Por las consideraciones anteriores, podemos observar que el currículo se construye a partir de un sistema de elementos estrechamente relacionados entre sí y posee, además, un carácter procesal (Gonzales et al., 1987). Es decir, ya sea para planificar o para desarrollar el currículo, existen elementos que de alguna manera orientan y otros que instrumentalizan el proceso educativo y sientan las bases para el desarrollo del currículo oficial. Esta propuesta teórica de análisis sirve de guía para el estudio de la Educación por el Arte en el currículo de la EBR en la Reforma Educativa de 1972 y permite conocer en profundidad los niveles, las bases y los elementos curriculares, así como el planteamiento pedagógico con el que se incluyeron las artes en el sistema educativo de la Reforma.

\section{La Reforma Educativa de 1972 y el currículo de Educación Básica}

Uno de los cambios más significativos y profundos en el sistema educativo peruano se produjo durante el período comprendido entre los años 1968 y 1975, durante el Gobierno Revolucionario de la Fuerza Armada (GRFA), al mando del general Juan Velasco Alvarado. A partir de la promulgación de la Ley General de Educación № 19326, en marzo de 1972 (Ministerio de Educación, Gobierno del Perú, 1972), en el Perú se iniciaría una de las reformas educativas más importantes no solo del país, sino de toda América Latina. 
La propuesta de reforma comenzó a partir del diagnóstico del sistema educacional elaborado por la Comisión de la Reforma Educativa a través de un Informe general. Esta comisión fue conformada en 1969 e incluía a más de 100 miembros que se desempeñaban como profesores, filósofos, sociólogos, antropólogos, psicólogos, economistas, músicos y arquitectos (Oliart, 2013), quienes fueron capaces de lograr una cooperación "como no había ocurrido nunca en el país y, probablemente en el mundo, atendiendo sólo a la capacidad, la honestidad y la vocación de servicio de los invitados" (Barrantes, 1989, p. 98).

El Informe general reveló que el sistema educativo, además de ser inadecuado para enfrentar los complejos problemas socioeconómicos del Perú, también había contribuido a perpetuar y agravar las desigualdades sociales, culturales y económicas de la sociedad peruana (Organización de las Naciones Unidas para la Educación, la Ciencia y la Cultura, 1976). Evidenció también que el sistema educacional mantenía el uso de ideas y modelos educativos extranjeros y descontextualizados con la realidad del país. Además de dichos aspectos, el diagnóstico enfatizó la problemática con respecto a lo siguiente: 1) un analfabetismo creciente; 2) la desatención de los niños de grupos sociales marginados; 3) la ausencia de un sistema de recuperación de la educación de los adultos; 4) la educación al servicio de una minoría; 5) una desconexión de la educación con la realidad del contexto peruano; 6) la falta de un sentido peruanista; 7) el intelectualismo, memorismo y una tendencia academizante en el enfoque educativo empleado; 8) una inadecuada formación y selección del magisterio; 9) rigidez, burocratismo y rutina en el sistema administrativo; y 10) una distorsión administrativa y financiera que afectaba a todo el sistema educativo (Ministerio de Educación, Gobierno del Perú, 1970).

Claramente, esta reforma no trataba únicamente de modificar el currículo y la organización educativa, sino de "darle un nuevo contenido a la educación y de reorientarla para la conquista de objetivos cualitativamente distintos a los que persiguiera en el pasado un ya obsoleto sistema educativo" (Velasco, como se citó en Oliart, 2013, p. 80). Aquellas insuficiencias y problemas encontrados debían corregirse, de manera tal que esta reforma educativa estuviera de acuerdo con el carácter y la naturaleza de las nuevas políticas del régimen.

De esta manera, la Reforma Educativa de 1972, además de introducir por primera vez el término currículum en el sistema educativo peruano, marca un hito en las propuestas educativas desde el Estado al interesarse por la inclusión de las tendencias pedagógicas e ideológicas progresistas imperantes en su época, por alcanzar el desarrollo de habilidades para el estudio crítico y por el empleo de métodos participativos de manera transversal a toda la propuesta curricular (Manrique, Revilla y Lamas, 2013/2014; Oliart, 2013). La Reforma se ve impregnada claramente por la búsqueda de una formación integral y por la intención de formar un nuevo tipo de individuo identificado con su contexto y con su propia cultura. De igual manera, considera que la educación es una vía por la cual el ser humano puede transferir las formas de la cultura a la que pertenece y es también el medio para capacitar a la persona para el trabajo, fomentando su participación activa en el proceso social para generar una transformación y un perfeccionamiento de la sociedad peruana (Ministerio de Educación, Gobierno del Perú, 1970). 
La estructura del sistema educativo de la Reforma comprende tres niveles: la Educación Inicial, la Educación Básica y la Educación Superior. La Educación Básica Regular (EBR) se plantea como segundo nivel educativo y eje del sistema; está destinada para todos los niños a partir de los seis años y hasta la adolescencia. Su carácter es general y obligatorio y se establece como el fundamento del desarrollo integral del educando (Ministerio de Educación, Gobierno del Perú, 1972). La EBR está compuesta por tres ciclos y nueve grados, los cuales pretenden cubrir de manera integrada los conocimientos, las actividades, la capacitación para el trabajo y la orientación del educando. Estas son llamadas áreas de acción educativa y están conformadas por diversas líneas de acción.

En la definición de la Estructura Curricular Básica de la Reforma, las líneas de acción educativa "son un conjunto de experiencias y contenidos, afines por su naturaleza, que permiten al educando desarrollar habilidades, destrezas, actividades, conocimientos y valores específicos que favorecen el descubrimiento y transformación de la realidad" (Ministerio de Educación, Gobierno del Perú, 1980, p. 10). Según el currículo de la Reforma, dentro de estas líneas de acción priman distintos aspectos: en algunas, mayormente los conocimientos o la capacitación para el trabajo; en otras, las actividades o la orientación del educando; y, en algunas otras, una combinación de varias de estas acciones.

Dentro de la acción educativa de Actividades, encontramos la inclusión de las artes. Estas aparecen como una línea de acción que integra las artes plásticas, la educación musical, la danza y la educación por el teatro, bajo la denominación de Educación por el Arte. De esta manera, el arte y la educación artística son considerados en esta reforma como fines valiosos en sí mismos y no solamente como un fenómeno superfluo y marginal que puede ser empleado para clases de "adorno" (Ministerio de Educación, Gobierno del Perú, 1970). Es decir, se considera que las artes son un aporte enriquecedor al currículo y que pueden marcar una diferencia en los aprendizajes y en el desarrollo de los educandos en comparación con el resto de las áreas de conocimiento que integran el currículo.

En otras palabras, la Educación por el Arte se presenta como una línea de acción fundamental que contribuye a la formación integral del individuo y a su desenvolvimiento pleno en la sociedad. Se reconoce que el enfoque de la línea dentro del currículo es, tal como su nombre lo indica, por las artes, es decir, uno en el que las artes se consideran un vehículo de aprendizaje de otros contenidos curriculares y un medio para lograr otros resultados educativos más generales (Bamford, 2009). Por tanto, la actividad artística debería estar presente en todas las acciones educativas y la creatividad debería ser el motor de todas las formas de comunicación y de realización pedagógica del sistema educativo.

\section{METODOLOGÍA}

La presente investigación es de tipo documental bajo un enfoque cualitativo, el cual, según Berg y Lune (2007), se refiere a los significados, conceptos, definiciones y descripciones de las cosas. En la misma línea, Gil (2002, como se citó en Figueiredo y Meurer, 2016, p. 521) afirma que la investigación documental es adecuada para los estudios que 
tratan sobre materiales que aún no recibieron tratamiento analítico. Precisamente, en la presente investigación, los documentos que serán las fuentes de análisis no han sido estudiados a nivel curricular desde el área de artes, por lo que resulta pertinente el desarrollo de la investigación bajo este paradigma. El método de investigación empleado es el método documental y se usa la técnica de análisis de documentos en párrafos y extractos de los textos oficiales publicados por el Ministerio de Educación, extraídos de acuerdo con los indicadores derivados de las categorías y subcategorías de análisis.

Un factor importante y decisivo en este tipo de investigación lo conforman los criterios de selección de las fuentes documentales, los cuales deben ser debidamente clarificados (Mogalakwe, 2006). En la presente investigación, los criterios tomados en cuenta han sido cuatro: 1) la naturaleza del documento, es decir, que sean documentos normativos oficiales; 2) la temporalidad, considerando que hayan sido expedidos en los años de la Reforma Educativa y durante su implementación (entre 1970 y 1980); 3) el emisor del documento, en este caso el Ministerio de Educación; y 4) su carácter público y de libre acceso.

Bajo dichos criterios, se recopilaron los siguientes documentos como fuentes de análisis: a) el Informe general de la Reforma Educativa (Ministerio de Educación, Gobierno del Perú, 1970); b) la Ley General de Educación № 19326 (Ministerio de Educación, Gobierno del Perú, 1972); c) el Currículum de Educación Básica Regular del Ministerio de Educación (Dirección General de Educación Básica Regular, Ministerio de Educación, 1972), d) la Estructura Curricular Básica del Ministerio de Educación (Ministerio de Educación, Gobierno del Perú, 1980)4; y e) las Guías metodológicas de Primero y Segundo Ciclo del Ministerio de Educación (Dirección General de Educación Inicial y Básica Regular, Ministerio de Educación, 1974). Tales documentos contienen tanto las bases curriculares para la Reforma como los fines de la educación, los aspectos curriculares esenciales para el desarrollo curricular, una orientación sobre acciones didácticas e integración de contenidos, entre otros aspectos por considerar en un análisis como el que se pretende realizar y que llevará a determinar las características curriculares de la línea de acción de Educación por el Arte de la Reforma Educativa de 1972.

Para el presente estudio, se han considerado como categorías de análisis los elementos curriculares de la Educación por el Arte en la EBR de la Reforma Educativa de 1972, entendidos como elementos orientadores e instrumentales (Gonzales et al., 1987). De igual manera, para visualizar las relaciones que existen entre dichos elementos y entre los niveles macro y microcurricular, se ha diseñado una propuesta teórica de análisis general del currículo, la cual también ha sido útil para orientar parte del análisis de esta investigación. A partir de cada elemento curricular, se han propuesto subcategorías que son analizadas y contrastadas con toda la propuesta educativa para desarrollar un panorama completo de la Educación por el Arte dentro del currículo reformado. A su vez, de estas subcategorías han surgido los indicadores que orientan el análisis documental.

\footnotetext{
${ }^{4}$ Edición reajustada de 1980. Esta es la única edición que nos pudo brindar el Ministerio de Educación del Gobierno del Perú.
} 
Se consideran, en concreto, dos categorías de los elementos curriculares: 1) los elementos orientadores de la Educación por el Arte con las subcategorías de diagnóstico, ideas rectoras, perfil y objetivos generales; y 2) los elementos instrumentales de la Educación por el Arte con las subcategorías de contenidos, objetivos específicos, actividades, recursos y evaluación.

Para el recojo de información, se diseñaron fichas individuales para cada subcategoría y cada documento de análisis, las cuales, además de detallar la cita textual o evidencia, especifican la página y sección del documento donde se localizó la cita, e incluyen un espacio para diversas observaciones. A estas fichas, al igual que a las categorías y subcategorías de análisis, se les asignó un código para clasificación y rápida localización durante el análisis.

El análisis de documentos se realizó por etapas y de manera sistemática. De acuerdo con Bowen (2009), el análisis de documentos implica leer superficialmente (revisión superficial), lectura (revisión a profundidad) e interpretación (p. 32). Por tanto, primero, se organizó la información en categorías relacionadas con el objetivo de investigación y se realizó una revisión superficial pero comprensiva de todas las fuentes documentales. Enseguida, se identificaron las citas textuales o evidencias que se consideraron significativas y relevantes, y se extrajo la información considerada pertinente para la investigación (Corbin y Strauss, 1990/2008). En esta parte del proceso, se añadieron las observaciones anotadas en las fichas que serían útiles para encontrar conceptos importantes relacionados con la investigación y que permitieran ubicar de manera rápida las subcategorías e indicadores. Posteriormente, se contrastaron los datos recogidos para encontrar la información nueva y la que era reiterativa.

En el momento inicial del análisis, más que hacer una relación de las citas textuales recogidas sobre el problema de investigación, se las interpretó a la luz de la teoría revisada y se les dio un significado de acuerdo con el elemento curricular al que pertenecen. Tal como indica Bowen (2009), los investigadores deben establecer el significado del documento y su contribución a los temas que están siendo explorados (p. 33). Posteriormente, se empleó una guía de análisis como estructurador conceptual, basada en la propuesta teórica de análisis general del currículo y desarrollada en el marco de la investigación, para establecer los aspectos teóricos de interés, para dar un orden y una secuencia lógica a la información y para considerar posibles categorías emergentes en el currículo.

De igual manera, se diseñaron matrices de consolidación y análisis, que fueron útiles para organizar los datos recogidos de algunos elementos que podrían tener vinculación con otros niveles del currículo. En dichas matrices, se ordenó la información por categorías y se revelaron evidencias a través de citas textuales vinculadas con otros elementos curriculares. En una columna adicional, se añadieron observaciones complementarias, en caso de que estas fuesen necesarias. Finalmente, se hizo una interpretación de la información para extraer las conclusiones más relevantes para los objetivos de la investigación.

\section{RESULTADOS Y ANÁLISIS}

El currículo de la Reforma presenta como elemento orientador un diagnóstico general de la educación en el que se abordan las problemáticas que aquejaron al sistema educativo hasta antes del currículo reformado. Dicho diagnóstico, publicado en el Informe general de la 
Reforma Educativa, y desarrollado en otros documentos curriculares oficiales, presenta un análisis de la realidad social, cultural y educativa, así como una sección dedicada al papel del arte en la educación en la que encontramos la problemática de la educación artística y de las artes en el sistema educativo. Aquí se advierte que el principal problema está relacionado con la poca importancia otorgada a las artes en el terreno educativo.

Es decir, el arte había sido marginado en el currículo hasta el grado de ser olvidado al interior de las escuelas. La primacía de los aspectos cognoscitivos, más aún bajo concepciones de la educación de corte memorista, terminaron por menoscabar el aporte de las artes a la formación y al desarrollo pleno del individuo. En consecuencia, el diagnóstico descubre un panorama hasta entonces incierto para la educación artística en el sistema educativo peruano, el cual se convierte en el punto de partida para la elaboración del nuevo currículo para la reforma de la Educación Básica.

En las fuentes documentales analizadas, se declara explícitamente que es preciso poner de relieve "la inspiración profundamente humanista y la vocación genuinamente democrática de la nueva educación" (Ministerio de Educación, Gobierno del Perú, 1972, p. 8). Este paradigma humanista se ve reflejado tanto en los elementos orientadores como en los elementos instrumentales del currículo y se convierte en una base fundamental para definir el perfil del individuo que se quiere formar a través del sistema educativo. En ese mismo sentido, en la Estructura Curricular Básica (Ministerio de Educación, Gobierno del Perú, 1980) se afirma que los elementos orientadores del currículo tienen su fundamento teórico en J. Piaget y R. Gagné, y que se orientan hacia el principio de una educación activa y centrada en el educando como un individuo libre y creador, bajo una concepción curricular integral y flexible.

A partir de esta premisa, se espera también que el currículo pueda ser adaptado a las necesidades del contexto. En términos precisos, en el apartado "Definición de Términos" de la Ley General de Educación № 19326, se precisa que "el curriculum es flexible cuando se adapta a las diferentes situaciones académicas del educando y permite a ésta optar entre diversas asignaturas y actividades educativas de acuerdo a su preferencia y vocación. Su opuesto es el curriculum rígido" (Ministerio de Educación, Gobierno del Perú, 1972, p. 89). La flexibilización del currículo no solo se plantea con respecto a los aprendizajes del educando, sino que se declara como un aspecto estructural que puede ser adecuado a las características de los propios niveles regional, zonal y comunal, y que puede ser adaptado de acuerdo con los valores y la realidad socioeconómica de la comunidad en la cual se ponga en práctica.

En lo que respecta a la concepción de un currículo integral, la Reforma plantea la importancia de contar con diversas experiencias de formación que contemplen la totalidad del individuo, de forma tal que logre desenvolverse plenamente y pueda desarrollar también a la sociedad a la que pertenece. Tales experiencias pretenden alcanzar el saber científico, comprender y vivir la actitud religiosa, percibir el arte y participar en la creación artística, practicar los principios éticos, valorar el trabajo y desarrollar destrezas en el campo laboral, así como comprometerse con la defensa de la integridad y el desarrollo de la realidad nacional. 
Adicionalmente, como sugerencia para el desarrollo del currículo y la integración de áreas educativas, los documentos de la Reforma plantean un enfoque metodológico que haga uso de los centros de interés, del aprendizaje basado en proyectos, de actividades generadoras y situaciones problemáticas, entre otros. Coincidentemente, todas estas son metodologías que están de acuerdo con la búsqueda de una formación activa, flexible e integral, y que promueven la interdisciplinariedad de las diversas áreas de acción educativa que se proponen dentro del currículo.

Con relación al rol del docente, la Dirección General de Educación Inicial y Básica Regular del Ministerio de Educación (1974) considera que el maestro es quien "crea el ambiente propicio a la actividad creadora, o el que la hace morir definitivamente" (p. 23), y que debería ser "un creador, un adulto sensible, que rodee al niño de experiencias ricas en percepciones, sentimientos e ideas y que las interrelaciones [sic] adecuadamente, para enriquecerlo permitiéndole un desarrollo feliz" (p.123). Es decir, el profesor, más que ser un guía u orientador para el desenvolvimiento artístico, es un impulsor del desarrollo de la creatividad, la sensibilidad y la imaginación de los alumnos. Su rol se configura como un elemento importante para generar ambientes que impulsen la participación activa del alumno en el proceso de enseñanza-aprendizaje. Por su parte, el educando se concibe como un ser que toma iniciativas, tiene opinión y moviliza su conciencia de ser participante (Dirección General de Educación Inicial y Básica Regular, Ministerio de Educación, 1974, p. 82), además de tener un espíritu crítico y reflexivo. Su lugar dentro del proceso educativo es crucial, ya que se considera como centro y agente principal, y como un movilizador de sus propios procesos de aprendizaje.

El tratamiento de las artes en los elementos orientadores del currículo de la Reforma es una pieza clave para comprender el enfoque que se encuentra detrás de la línea de acción de Educación por el Arte. En ellos se declara explícitamente que la actividad artística es una parte esencial de la educación de todo hombre y que las artes poseen un lugar en el currículo no solamente para cumplir una función auxiliar en el estudio de otras materias mediante experiencias integradoras con los lenguajes artísticos, sino para desarrollar también el sentido artístico y estético en el educando, así como sus capacidades creadoras, expresivas e imaginativas, de modo que se rescata el valor de las artes por sí mismas en la educación. La Educación por el Arte se encuentra, por tanto, dentro del enfoque por las artes, pero con características evidentes de un enfoque para las artes.

Esta línea de acción se consolida en el currículo como una que aporta experiencias fundamentales para el desarrollo integral del individuo y que potencia las capacidades psicomotrices, afectivo-sociales y cognitivas con - y a través de- las artes. Esta búsqueda de formación integral es, en realidad, el elemento rector de los objetivos de la EBR, por lo que la línea satisface dicha búsqueda mediante las experiencias con las artes y al abarcar también los aspectos intelectuales, físicos, emocionales y sociales del niño.

Con relación al nivel microcurricular y a sus elementos instrumentales, se pudo observar que los contenidos de las disciplinas que componen la Educación por el Arte tienen en común los siguientes elementos: a) la expresión a través de la disciplina artística, b) el 
contacto y la apreciación de otras expresiones artísticas en el ámbito nacional, c) la experimentación y la creación artística, y d) el desarrollo de la percepción de todas las formas de arte. Las evidencias en los documentos demuestran que las disciplinas artísticas pueden conjugarse con la finalidad de crear experiencias más complejas para desarrollar los contenidos y lograr los objetivos de la línea de acción. Algunos ejemplos se advierten en estas citas: "[El educando] Realiza creaciones de expresión corporal originadas por motivaciones sonoras” (Dirección General de Educación Básica Regular, Ministerio de Educación, 1972, p. 58), "[El educando] Dramatiza sus canciones en el aula dividiéndose responsabilidades (...) las que serán asumidas rotativamente" (Dirección General de Educación Básica Regular, Ministerio de Educación, 1972, p. 37), “[Para realizar dactilopintura] Motivación: actividades de expresión corporal o estímulos musicales" (Dirección General de Educación Inicial y Básica Regular, Ministerio de Educación, 1974, p. 153), "Pintar con estímulo musical: (...) se les indica [a los educandos] que procuren seguir el ritmo pintando con sus dedos" (Dirección General de Educación Inicial y Básica Regular, Ministerio de Educación, 1974, p. 135).

Los objetivos específicos de la Educación por el Arte son precisados en las disciplinas artísticas que integran dicha línea de acción, como el teatro, la música, la danza y las artes plásticas, y pretenden que el educando pueda conocer sus posibilidades expresivas y que adquiera un mayor dominio de ellas, de manera tal que pueda exteriorizar lo que siente, percibe y piensa de sí mismo y del mundo que lo rodea. Las evidencias indican que no solo se espera el desarrollo de la expresión, sino también la apreciación y comprensión de otros tipos de expresión artística, especialmente los relacionados con la expresión artística nacional, ya sea musical, corporal o plástica.

Con finalidad de indagar sobre relaciones que se dan entre el nivel macro y microcurricular, en este elemento instrumental se emprendió un análisis adicional relacionando los objetivos específicos con los elementos orientadores del currículo. Con ese propósito, se analizaron los objetivos específicos a la luz del diagnóstico educativo presentado en el Informe general y se encontró que, en la Educación por el Arte, se emplean explícitamente estrategias que contrarrestan las deficiencias encontradas en el sistema educativo previo a la Reforma. Esto demuestra que se realizó un análisis a conciencia de las necesidades y de las posibles soluciones que podían plantearse en el currículo reformado. Todas estas soluciones llevan hacia un currículo que permita acabar con el enfoque memorista y mecánico, y que plantea una educación que promueve la expresión y la creatividad a través de los diversos lenguajes artísticos.

En lo que respecta a las actividades, el análisis reveló aspectos interdisciplinarios de la Educación por el Arte en relación con el resto de las líneas de acción educativa. Un ejemplo se observa en la línea de Lenguaje, en la cual se emplean actividades de discriminación visual y auditiva, juegos con colores y con sonidos, y representaciones plásticas para desarrollar los contenidos de dicha materia (véase la Figura 2). Por su parte, las recomendaciones de actividades para Ciencias Naturales son los juegos con colores y sonidos, mientras que para Ciencias Sociales se sugiere "Realizar representaciones plásticas de los grupos humanos con los que se relacionan y de los lugares visitados” (Dirección General de Educación Básica Regular, Ministerio de Educación, 1972, p. 33). Estas actividades con cualidades interdisciplinarias son las que le dan el sentido a la inclusión de las artes en la educación bajo 
el enfoque empleado por la Reforma, en el que son consideradas un medio, más que un fin en sí mismas.

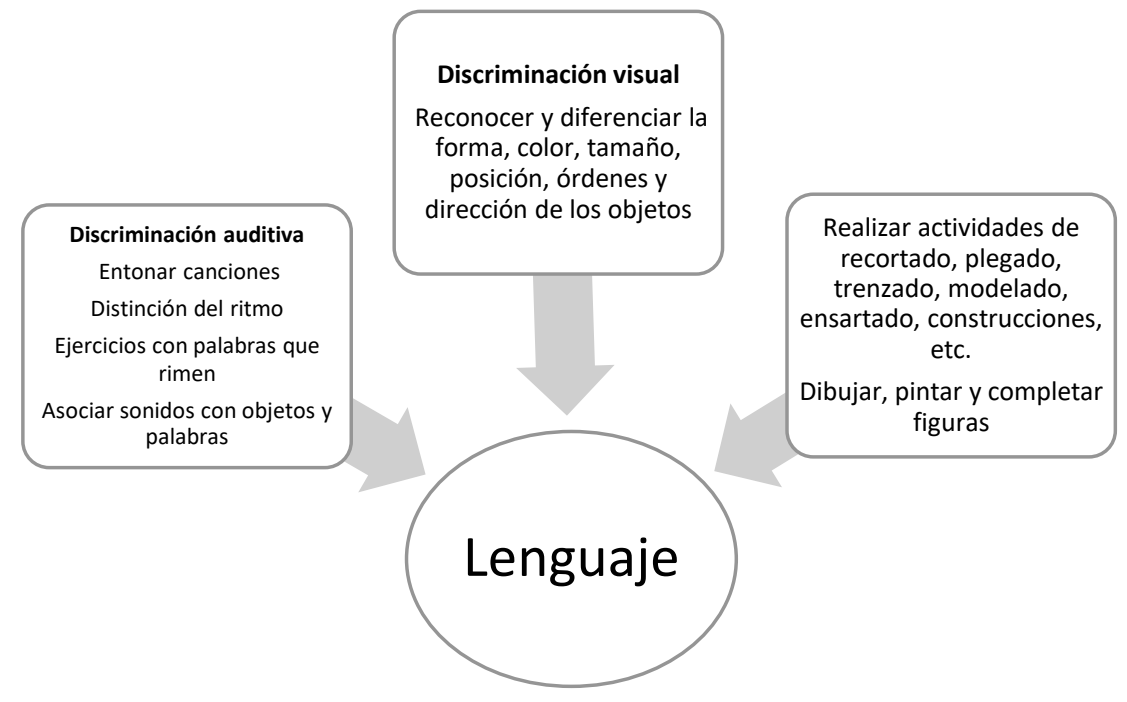

Figura 2. Actividades de Educación por el Arte empleadas en el área de Lenguaje

Adaptada de Currículum de Educación Básica Regular, por Dirección General de Educación Básica Regular, Ministerio de Educación, 1972, Minedu, pp. 26-27.

Con relación a la evaluación, en los planteamientos de la Reforma se espera que el profesor no emita juicios evaluativos ni realice críticas con respecto al trabajo creativo desarrollado por el niño en cualquiera de las disciplinas artísticas. Tal es el caso del área de Teatro, en la cual se menciona que el profesor "No emite juicios evaluativos ni propone modelos, solamente estimula, pero estimula creativamente, con alegría, interés y amistad" (Dirección General de Educación Inicial y Básica Regular, Ministerio de Educación, 1974, p. 81). Mientras, en Música se hace énfasis en lo siguiente: "Durante los comentarios de las audiciones el profesor se abstendrá de: Emitir su opinión personal [o de] Calificar lo escuchado" (Dirección General de Educación Inicial y Básica Regular, Ministerio de Educación, 1974, p. 114). En ambos casos, el docente no puede calificar o evaluar al niño, sino solo incentivar su creatividad. Por su parte, en Artes Plásticas, se lee lo siguiente: "EL PROFESOR DEBE EVITAR: Medir con patrones de adultos el trabajo de los niños, calificando el resultado de bonito o feo. Corregir y criticar el trabajo infantil. Comparar el trabajo de un niño con el otro" (Dirección General de Educación Inicial y Básica Regular, Ministerio de Educación, 1974, p. 122).

Así, el planteamiento de la evaluación de la Educación por el Arte podría resumirse en esta afirmación del currículo: "La experiencia del arte debe ser una parte feliz del día escolar y una oportunidad para resolver problemas que no tienen que ver [con] las respuestas 'correctas' o 'incorrectas', sino con el pensamiento y la respuesta creadores" (Dirección General de Educación Inicial y Básica Regular, Ministerio de Educación, 1974, p. 121). Se puede observar, entonces, que la evaluación se asocia con la emisión de juicios sobre el trabajo del niño, la medición de su actuación con relación al otro, y la corrección y crítica 
permanente de su quehacer con las artes. Con respecto al profesor, se identificó que su papel no es el de evaluar ni calificar alguna de las actividades artísticas que desarrollan los alumnos y que debe evitar emitir opiniones sobre el desarrollo del educando en el proceso educativo.

En este aspecto, llama la atención que en la Educación por el Arte no se plantee alguna forma complementaria de valorar el desarrollo del niño en su relación con las artes. Concordamos con Eisner (2002/2004) cuando afirma que evitar cualquier acercamiento con la valoración de los procesos educativos implica tergiversar la evaluación y "supone renunciar a la responsabilidad profesional del trabajo" (p. 220). Es decir, al abandonar la evaluación, el profesor abandona también la oportunidad de conocer las consecuencias de su enseñanza y de mejorar sus prácticas educativas, así como de abrir la posibilidad de orientar al alumno en su proceso de aprendizaje (Eisner, 2002/2004; Ruiz, 2005/2013). Entonces, la evaluación en la Educación por el Arte bien podría manejarse no para comparar o calificar al educando, sino para crear condiciones para un desarrollo pleno en el niño, para reconocer las diferencias entre las expresiones de los alumnos y para favorecer y estimular la diversidad creativa (Frega y González, 2010).

\section{CONCLUSIONES}

Se puede concluir que, en la Reforma Educativa peruana de 1972, la dimensión macrocurricular se sitúa explícitamente bajo el paradigma filosófico humanista, fundamentado en las teorías de J. Piaget y R. Gagné, y bajo una concepción curricular integral y flexible, que tiene como agente principal de la educación al mismo educando. En este nivel, se declara que la actividad artística es una parte esencial de la educación de todo hombre y que las artes poseen un lugar en el currículo, no solamente para cumplir una función auxiliar en el estudio de otras materias mediante experiencias integradoras con los lenguajes artísticos, sino para desarrollar también el sentido artístico y estético en el individuo, así como sus capacidades creadoras, expresivas e imaginativas, lo que rescata el valor de las artes por sí mismas en la educación. Se encuentran, por tanto, dentro del enfoque por las artes, pero con características evidentes de un enfoque para las artes.

En lo que respecta al nivel microcurricular, este responde directamente a las orientaciones del nivel macrocurricular y cuenta con un enfoque interdisciplinario para los procesos de enseñanza-aprendizaje de las disciplinas artísticas que integran la Educación por el Arte (teatro, música, artes plásticas y danza) con el resto de las líneas de acción del currículo. El sentido de inclusión de las artes bajo el enfoque por las artes se hace evidente en el planteamiento de actividades de artes plásticas, música y teatro para el desarrollo de los contenidos con otras líneas de acción educativa del currículo tales como Lenguaje, Ciencias Naturales y Ciencias Sociales.

En suma, la Reforma Educativa peruana de 1972 hace visible la importancia de las artes para una educación que fomente el aprendizaje a lo largo de la vida, enfatiza en el aporte de las artes en la solución de problemas sociales y culturales, y las considera un elemento fundamental para la construcción de la identidad cultural. Finalmente, la línea de acción de 
Educación por el Arte propone un conjunto de experiencias que potencian las capacidades cognitivas, motoras y afectivo-sociales del educando en todas las otras áreas de conocimiento del currículo y le permiten explorar, imaginar, expresar, comunicar y crear con - y a través de-las artes, y alcanzar así el desarrollo integral del educando. Estas características la hacen vigente hoy en día y reflejan el carácter visionario e innovador que esta propuesta tuvo en su momento. 


\section{REFERENCIAS BIBLIOGRÁFICAS}

Bamford, A. (2009). El factor ¡WUAU! El papel de las artes en la educación. Barcelona: Octaedro.

Barrantes, E. (1989). Historia de la educación en el Perú. Lima: Mosca Azul Editores.

Berg, B. L. y Lune, H. (2007). Qualitative research methods for the social sciences (6. $\frac{\text { a }}{\text { ed.). }}$ USA: Pearson.

Bowen, G. (2009). Document analysis as qualitative research method. Qualitative research Journal, 9(2), 27-40. doi:10.3316/QRJ0902027

Cantón Mayo, I. y Pino-Juste, M. (Coords.). (2011). Diseño y desarrollo del currículum. Madrid: Alianza Editorial.

Corbin, J. y Strauss, A. (2008). Basics of qualitative research: Techniques and procedures for developing grounded theory (3. $\underline{a}$ ed.). California: Sage Publications. doi: $10.4135 / 9781452230153$ (Trabajo original publicado en 1990)

Díaz Bazo, C., Persico, R., Del Mastro, C. y Ferrazzi, G. (1992). Desarrollo del currículo de los centros educativos: análisis de los elementos orientadores e instrumentales. Educación, 1(1), 77-84. $\quad$ Recuperado de http://revistas.pucp.edu.pe/index.php/educacion/article/view/4402

Dirección General de Educación Básica Regular, Ministerio de Educación. (1972). Currículum de Educación Básica Regular. Lima: Minedu.

Dirección General de Educación Inicial y Básica Regular, Ministerio de Educación. (1974). Guías metodológicas de Primero y Segundo Ciclo. Lima: Minedu.

Eisner, E. (2004). El arte y la creación de la mente: el papel de las artes visuales en la transformación de la conciencia (Trad. G. Sánchez Barberán). Barcelona: Paidós. (Trabajo original publicado en 2002)

Figueiredo, S. y Meurer, R. (2016). Educação musical no currículo escolar: uma análise dos impactos da Lei no 11.769/08. OPUS, 22(2), 515-542. doi:10.20504/opus2016b2220.

Frega, A. y González, G. (Comps.). (2010). Evaluación de aprendizajes musicales. Buenos Aires: Bonum.

Gimeno Sacristán, J. (1988). El curriculum: una reflexión sobre la práctica. Madrid: Morata.

Gimeno Sacristán, J. y Pérez Gómez, A. I. (1992). Comprender y transformar la enseñanza. Madrid: Morata. 
Giráldez, A. y Palacios, A. (2014). Educación Artística en Iberoamérica. Informe. Madrid: Organización de Estados Iberoamericanos.

Gonzales, R., García, C., Saco, R. y Reátegui, N. (1987). Lineamientos básicos para el trabajo curricular. Documento de trabajo. Lima: CISE-PUCP.

Jiménez, L., Aguirre, I. y Pimentel, L. G. (Coords.). (2009). Educación artística, cultura y ciudadanía. Madrid: OEI.

Manrique, L., Revilla, D. y Lamas, P. (2014). Theoretical approaches underlying Primary Education curricula in Peru. En W. F. Pinar (Ed.), International handbook of curriculum research (2. ${ }^{\mathrm{a}}$ ed., pp. 376-390). Nueva York: Routledge - Taylor \& Francis Group. (Trabajo original publicado en 2013)

Ministerio de Educación, Gobierno del Perú. (1970). Reforma de la Educación Peruana: Informe general. Lima: Minedu.

Ministerio de Educación, Gobierno del Perú. (21 de marzo de 1972). Ley General de Educación № 19326. Decreto de Ley. Lima: Minedu.

Ministerio de Educación, Gobierno del Perú. (1980). Estructura Curricular Básica. Lima: Minedu.

Mogalakwe, M. (2006). The use of documentary research methods in social research. African Sociological Review, 10(1), 221-230. Recuperado de http://citeseerx.ist.psu.edu/viewdoc/download?doi=10.1.1.454.5260\&rep=rep1\&ty pe $=$ pdf

Oliart, P. (2013). Educar en tiempos de cambio, 1968-1975 (Vol. 13). Lima: Derrama Magisterial.

Organización de Estados Iberoamericanos para la Educación, la Ciencia y la Cultura. (2014). Miradas sobre la educación en Iberoamérica 2014. Avances en las Metas Educativas 2021. Recuperado de https://oei.int/publicaciones/miradas-sobre-la-educacion-eniberoamerica-2014-avances-en-las-metas-educativas-2021

Organización de las Naciones Unidas para la Educación, la Ciencia y la Cultura. (1976). La reforma de la educación en Perú. París: Unesco.

Organización de las Naciones Unidas para la Educación, la Ciencia y la Cultura. (2006). Hoja de Ruta para la Educación Artística. Lisboa: Unesco. Recuperado de http://www.unesco.org/new/fileadmin/MULTIMEDIA/HQ/CLT/CLT/pdf/Arts_Edu _RoadMap_es.pdf 
Revista Enfoques Educacionales - Año 2021 - Vol. 18: 4-23

Ruiz, J. M. (2013). Teoría del currículum: diseño, desarrollo e innovación curricular. Madrid: Editorial Universitas. (Trabajo original publicado en 2005) 